\title{
Ceramic dies selection for electrical resistance sintering of metallic materials
}

\author{
F.G. Cuevas ${ }^{1}$, D. Andreouli ${ }^{2}$, J.M. Gallardo ${ }^{3}$, V. Oikonomou ${ }^{2}$, J. Cintas ${ }^{3}$, Y. Torres ${ }^{3}$, J.M. \\ Montes $^{3}$
}

${ }^{1}$ Department of Chemical Engineering, Physical Chemistry and Materials Science, Escuela Técnica Superior de Ingeniería, Universidad de Huelva, Campus El Carmen, Avda. Tres de Marzo s/n, 21071 Huelva, Spain

${ }^{2}$ MIRTEC (Materials Industrial Research \& Technology Center), 72nd km of AthensLamia National Road, P.O. Box 18646, 34100, Chalkida, Greece

${ }^{3}$ Metallurgy and Materials Engineering Group, Escuela Técnica Superior de Ingeniería, Universidad de Sevilla, Camino de los Descubrimientos, s/n, 41092 Sevilla, Spain

Corresponding author: F.G. Cuevas, e-mail: fgcuevas@dqcm.uhu.es Tel.: +34959217448

\begin{abstract}
Processing metallic powders by electrical resistance sintering requires the use of insulating ceramics dies. Selecting the appropriate ceramic material according to the electrical, thermal and mechanical properties is a need. Dies produced with several ceramic materials have been tested during the production of cemented carbide in order to check their behaviour in the process and final product properties. Tialite/mullite, zircon/mullite, zirconium phosphate based ceramic, yttria-stabilized zirconia and sialon, in most cases with modified compositions and shaping processes in order to achieve a high density, have been tested. Dry powder processing by cold isostatic pressing and furnace sintering resulted to be the better process for dies production. The effect of die properties on the produced cemented carbide, and the behaviour and life of the die during the production have been analysed. Very smooth die surface increases the number of cycles withstood during metallic parts production, because of lower extraction stresses, as checked for sialon dies. Zirconium phosphate based dies, with low thermal conductivity, show the most densified hard metal parts surface.
\end{abstract}

KEYWORDS: ceramic materials; insulating dies; electrical resistance sintering; cemented carbide

\section{INTRODUCTION}

Appropriate materials selection is a key aspect for ....... [1-3]. Improvements in the properties of the selected material usually lead to significant economic savings during the use of the produced parts. This material selection does not only apply to the final products, but also to the different materials involved in their production. Thus, when the equipment necessary to obtain the final product is susceptible of being a problems source, its adequate selection can be as critical as the properties of the final product itself. One such case is the fabrication of cemented carbides.

Hard metal or cemented carbide is the name given to a mixture of tungsten carbide (WC) and a binder ..... (Co) [4-5], although other compositions are also possible. The resulting composite, with composition usually tailored to the desired application, is a very hard material used for the production of cutting tools, as drills, saws or inserts, needed for processing metallic materials, or geo-engineering [6]. The resulting product shows, in general, better global behaviour than other options as high-speed steels. 
The production of these cemented carbides is usually carried out by a compaction process into a specific form, a green part, that is then furnace sintered to obtain the final part. During sintering, the binder partially melts, surrounding the carbides that remain in solid state at the sintering temperatures. The obtained metal matrix composite is additionally grinded and polished to the final shape [7]. A new technique for the production of cemented carbides, based on sintering the pressed powders mixture by leading an electrical current through it (Joule effect), has been recently studied [8]. This technique, known as electrical resistance sintering (ERS), is one of the modalities of the known as Field Assisted Sintering Techniques [9], having the possibility of restraining grain growth and therefore improving properties. During ERS, powders are placed into an isolating die, and two conductive electrodes/punches act to exert the desired pressure to compact the powders, at the time that a high intensity current, of up to approximately ......, with a low voltage of up to $10 \mathrm{~V}$, passes through the powder mass. These features are easily satisfied by adapting a spot welding machine to carry out the ERS process $[10,11]$.

A very important factor that affects the electrical consolidation is the selection of the die material. The die material has to be good electrical and thermal insulator, being resistant to thermal shocks, chemically inert, and having good mechanical and wear properties. This will make the electric current passing through the metallic powders, avoiding heat leaks and a more efficient heating and sintering of the cemented carbides. In addition, having the adequate roughness for de-moulding will decrease wear, and a good mechanical resistance will help resisting stresses. This makes the selection of the appropriate die material a key point in the development of this novel cemented carbides processing. Requirements for the ceramic dies were set based on bibliography and previous experience as follows: flexural strength higher than ...., thermal expansion lower than ....., electrical resistivity higher than .... and refractoriness (temperatures higher than $1000^{\circ} \mathrm{C}$ ).

According to the literature [12-31], the following refractory oxide materials with attractive properties can be considered: aluminium ..................... These advanced ceramics exhibit excellent properties and are used in high temperature and very demanding applications: metallurgical industry, glass industry, electronics, thermal barrier coatings and thermal shock resistant, high temperature fuel cells, oxygen detectors, catalysts supports, wall flow filters for diesel particulate emission control in automotive industry, etc. However, alumina does not withstand thermal shocks, and cordierite has a maximum service temperature close to ....., therefore not being considered for the current application. The materials with properties fulfilling most of the high demanding requirements set above are mainly:

In this paper, several ceramic materials have been tested in order to establish their benefits and drawbacks in the ERS process. Some of these ceramics have been specifically prepared for this application, whereas other ones are commercial ceramics. Among the many possible available compositions and production techniques, an important goal was always considered during dies production. Taking into account that the durability of the ceramic dies is limited, relatively cheap materials and production 
techniques were selected. Therefore, considering some basic properties of candidate ceramics, several different ceramics and production techniques have been tested.

\section{CONCLUSIONS}

After the analysis of experiments results, conclusions can be summarized as follows:

- Several ceramic materials have been tested as dies during ERS experiments with WC6 Co powders. Tialite/mullite, zircon/mullite, zirconium phosphate based ceramic, yttriastabilized zirconia and sialon, in general after conveniently modifying the original composition in order to achieve a low porosity, were studied. Porosities among ........were achieved.

- The produced dies had to be reinforced by wrapping with a metallic rim. Shrink fitting process is of high importance as can induce cracking in the dies. Failure extension from mouth cracks occurs after a conical clearance should be prepared near the tube ends. Also machining and polishing should improve their behaviour. Initial roughness Ra of dies ranges from ......... Dies roughness do not increase steadily till die failure. In several cases roughness did not increase at all before failure.

- 37 dies have been tested in this work, with about 140 samples processed. Experiences were carried out using .... and current passing time of 8YSZ, dies withstand progressively more ERS cycles before failure. Failure seems not to be related to the ERS action, but mainly to the .stresses, increasing as additional cycles are carried out except for Samples do freely came out of die, with a very smooth surface expected for a commercial product.

- The lower thermal conductivity of loses, improving the densification of the cemented carbides, whereas commercial dies lead to less dense metallic parts exhibiting a porous outer layer.

Therefore, by improving the roughness of the material with polishing and carefully wrapping the die with a metallic rim, ceramic could be the best candidate material for effective ERS sintering. 